\title{
HISTORIA Y DESARROLLO DEL MODELO ANDALUZ DE ARQUEOLOGÍA
}

\author{
HISTORY AND DEVELOPMENT \\ OF THE ANDALUSIAN MODEL \\ OF ARCHAELOGY
}

\section{VICENTE SALVATIERRA CUENCA (*)}

\section{RESUMEN}

En este artículo se hace un análisis de la actuación político-administrativa, en arqueología, del Gobierno Autónomo de Andalucía, desde la consolidación del nuevo sistema político hasta la actualidad. Se defiende la idea de que en 1985 se creó un Modelo de Intervención sumamente original, que ha sufrido en los últimos años tales transformaciones, que puede hablarse de que hoy existe un nuevo Modelo radicalmente diferente. La relevancia del primero deriva de varias de sus características y de los mecanismos utilizados para su elaboración, muy distintos de los empleados en otras Comunidades Autónomas. Y el conjunto del proceso tiene interés porque es fruto de la existencia en Andalucía, al menos hasta ahora, de la decisión de llevar a cabo una política arqueológica, y no tratar el tema sólo desde el punto de vista administrativo, y por aplicarse en la Comunidad mas extensa y posiblemente de mayor riqueza arqueológica de España.

(*) Area de Historia Medieval. Departamento de Territorio y Patrimonio Histórico. Facultad de Humanidades. Universidad de Jaén. 23071 Jaén.

$\mathrm{El}$ artículo fue remitido en su versión final el 12-IV-94.

\begin{abstract}
This paper analyses the administrative policy for Archaeology carried out by the Regional Government in Andalucía (Junta de Andalucía) from the establishment of the new political system up to now. A higly original archaeological policy was created in 1985; however, such a policy has changed in recent years to the extent of becoming a completely different one. The initial policy was particularly important on account of some of its characteristics and the mechanisms used to carry it out, and it was unique when compared to the policies in other administrative regions in Spain. The evolution of this policy is worth attention, not only because it springs out of the interest in carrying out an archaeological strategy in Andalucia instead of dealing with the issue from a purely administrative point of view, but also because it affects the region with the biggest and richest archaeological potential in Spain.
\end{abstract}

Palabras clave: Modelo Andaluz de Arqueología. Política Arqueológica. Arqueología Andaluza. Gestión en arqueología.

Key words: Archaeological Model in Andalucía. Archaeological Policy. Andalusian archaeology. Archaeological management 


\section{INTRODUCCION}

A finales de los años sesenta y principios de los setenta se inicia la transformación ideológica del sistema político instaurado por Franco, como correlato de los cambios materiales e ideológicos producidos en la propia sociedad española.

La desaparición de la dictadura y la instauración de la democracia (1975-78) fue seguida a mediados de los ochenta por una transformación en profundidad de la estructura administrativa del Estado. Frente al fuerte centralismo existente hasta ese momento, se crea lo que se ha denominado el "Sistema de las Autonomías". El territorio se divide en 17 Comunidades Autónomas, de muy distinto tamaño y población, desde la mayor, Andalucía, que abarca las ocho provincias del Sur, hasta Comunidades con una sóla provincia como Murcia o Navarra, pero todas con gobiernos y parlamentos propios. No todas tienen las mismas competencias, existiendo al menos dos "niveles". Uno con "competencias plenas" formado por las "nacionalidades históricas" es decir, aquellas que durante la II República española (1933-1936) tuvieron un Estatuto de Autonomía propio: Cataluña, País Vasco y Galicia, más Andalucía, donde se aprobó por referendum especial celebrado en 1980 . Y otro por el resto.

A partir de este momento el tratamiento de los problemas que plantea la arqueología (desde la investigación a la conservación) y los modelos de intervención (legislación, gestión, normas para la realización de actuaciones arqueológicas, etc.) se enfocan de forma diferente en cada una de las 17 Comunidades en función de las competencias transferidas, de los recursos económicos disponibles y del interés de los gobiernos autónomos en el tema, que difiere considerablemente de unos a otros, incluso entre los de Comunidades gobernadas por el mismo partido político (1).

En este marco, una de las razones para estudiar lo sucedido en el campo de la arqueología en Andalucía deriva precisamente de su gran

(1) Ninguno de los partidos políticos españoles tiene en realidad una política definida o mínimamente coherente en materia de Patrimonio Histórico, lo que explica la escasa incidencia que este tema tiene en la política española. De esta forma, la acción individual de los responsables del área en cada Comunidad tuvo en los primeros años una importancia decisiva en el diseño de cada modelo. tamaño, y de ser una de las zonas más ricas en yacimientos (Rodríguez y otros, 1993) al combinarse extensión, territorios costeros y tierras muy fértiles.

\section{La arqueología en España antes del modelo autonómico}

El inicio del cambio político o "primera fase de la transición" (1975-1978) coincide con un aumento en el número de arqueólogos integrados en las diversas universidades y demás instituciones científicas españolas y con un cambio en la propia orientación de la disciplina, todo lo cual afectó de forma importante, aunque distinta en sus resultados, a sus divisiones temporales (Ruíz, Molinos y Hornos, 1986; Martínez Navarrete, 1989; Salvatierra, 1990).

La "segunda fase de la transición" es el periodo que va desde la aprobación de la Constitución en diciembre de 1978, al establecimiento del régimen de las autonomías. Políticamente el periodo corresponde a los gobiernos conservadores de UCD y al primer gobierno del PSOE. Su duración fue muy desigual en cada una de las Comunidades en lo que se refiere a la aprobación de los Estatutos de Autonomía y al traspaso de competencias (2). Se trata de un periodo muy complejo, en el que la normativa general central fue dejando paso a la autonómica, con las contradicciones inherentes a tal situación.

La Dirección General de Bellas Artes, organismo responsable de la arqueología en el gobierno central, llevó a cabo una política de primar la investigación individualizada, al igual que había sucedido en las épocas anteriores. En el plano de la gestión puede observarse cierta organización de las actividades de urgencia, alentándose la realización de estas en todo el territorio, aunque sin crear un cuerpo especializado y sin que esa política fuese acompañada de una adecuada dotación económica. La arqueología urbana aparece por primera vez con cierta consistencia y mínima dotación económica ( $A r$ queología de las ciudades, 1985). No hubo virtualmente una política de conservación o res-

(2) Los primeros Estatutos aprobados fueron los de Cataluña y País Vasco en 1979 y el último el de Castilla-La Mancha en febrero de 1983. En lo que se refiere a Andalucía la aprobación del Estatuto se produjo en diciembre de 1981, mientras que el traspaso de las competencias en materia de arqueología y patrimonio no se completó hasta 1984. Desde el primer momento el gobierno andaluz estuvo en manos del PSOE, con mayoría absoluta en todas las legislaturas. 
tauración. Finalmente, la difusión, se redujo a la colaboración en la financiación de algún congreso y al mantenimiento de una revista oficial de aparición irregular (Noticiario Arqueológico Hispánico), con lo que la publicación de los resultados de la investigación que se realiza será muy parcial y tardía.

No obstante, antes de la obtención de competencias en arqueología se producen diversas iniciativas de otros organismos político-administrativos (3) y de algunas universidades, tanto en la financiación de actividades como en el campo de la difusión, en este con publicaciones (Primeras jornadas, 1983; Arqueología del País Valenciano, 1985; Arqueología en Alicante, 1986 y Homenaje, 1986) que hasta cierto punto pueden considerarse como precursoras o puntos de partida de las Memorias que en la actualidad publican las Comunidades Autónomas, Diputaciones o Ayuntamientos. La arqueología urbana tendrá una presencia importante, especialmente en algunas poblaciones como Zaragoza (Arqueología urbana, 1986; La Plaza de la Seo, 1989) y Tarragona (Els enterraments, 1987), auténticas precursoras en este campo, con independencia de la situación actual por la que atraviesan.

Teniendo en cuenta los pobres puntos de partida, los cambios experimentados en todo el país han sido en consecuencia muy profundos, aunque la política arqueológica de las Comunidades Autónomas ha diferido mucho de unas a otras. A este respecto empiezan a aparecer descripciones y análisis de gran interés sobre los "modelos" y problemas de algunas Comunidades (Velasco, 1992; Blasco y Valle, 1992; Domínguez y otros, 1994; Acién, 1992) y Ayuntamientos (Peral, 1994; Riera, 1994). Por lo que se refiere a Andalucía, debe distinguirse entre el proyecto general de intervención sobre el Patrimonio Histórico (Plan General de Bienes Culturales) y las líneas específicas del tratamiento de la arqueología (Modelo Andaluz de Arqueología, desde ahora MAA).

\section{EL PLAN GENERAL DE BIENES CULTURALES DE ANDALUCÍA}

En 1987 la Dirección General de Bellas Artes (luego de Bienes Culturales) empezó a tra-

(3) La estructura político-administrativa queda formada en estos momentos además de por los organismos centrales, por el Gobierno Autónomo a nivel regional, las Diputaciones a nivel de la provincia y los Ayuntamientos a nivel local. bajar en la creación de una política global, un proyecto estratégico, creándose el Plan General de Bienes Culturales, que fue aprobado en 1989 por todos los grupos del Parlamento Andaluz.

El Plan contemplaba cuatro campos: Etnología, Arqueología, Bienes Muebles y Bienes Inmuebles (4), sobre los que debía actuarse en cinco grandes líneas: Investigación, Restauración, Conservación, Protección y Difusión. Por otro lado, la propia concepción del Plan sugería que los distintos campos debían interrelacionarse, pero no se preveían mecanismos para lograrlo. Así por ejemplo, nada garantizaba la presencia de la arqueología durante la intervención en los Bienes Inmuebles.

No obstante, es preciso advertir que se empezó a trabajar en la preparación del Plan General después de que en algunos campos hubiese comenzado a aplicarse una política concreta, caso de la arqueología, para la que desde 1985 venía desarrollándose un Modelo de intervención de rasgos originales. El Plan lo que hizo fue incorporar esos elementos, sin establecer realmente fórmulas de integración, lo que quizá esté en el origen de algunas de sus disfunciones y contradicciones.

Las dificultades debían salvarse gracias a la estructura administrativa inicial, basada en que en cada provincia existía una única unidad de Bienes Culturales en la que estaban integrados el arqueólogo, el arquitecto y el historiador del arte, que en teoría debían trabajar coordinados (5), en vez de separarlos en departamentos casi estancos como sucede sobre todo en el modelo italiano. En la realidad, la colaboración parece haber sido mas bien escasa y cada uno de los campos ha tenido un desarrollo autónomo, aunque muy desigual. No entraremos ahora en cada uno de ellos aunque en determinados aspectos, sean inseparables de la arqueología.

\section{EL MODELO ANDALUZ DE ARQUEOLOGÍA}

Se trata de un sistema cuyo punto de partida se encuentra en las experiencias italianas, aun-

(4) Campos recogidos en la Ley 16/1985 de 25 de junio del Patrimonio Histórico Español, desarrollada a su vez en algunos aspectos importantes por la propia ley del Patrimonio Histórico de Andalucía (Ley 1/1991 de 3 de julio). Los bienes bibliográficos y documentales, contemplados también en la ley, tienen un tratamiento específico

(5) Los etnólogos prácticamente no tenían papel en ese primer desarrollo. 
que ha estado muy lejos de copiarlas. El MAA está compuesto por el conjunto de estructuras de muy diferente tipo (ejecutivas, administrativas, consultivas) creadas para la intervención; pero en cierta forma también son inherentes al mismo las decisiones concretas que se tomaron para desarrollar la intervención y el propio sistema de llegar a tales decisiones. Siempre se consideró que el marco nunca estaría completo y terminado, sino que estaba sujeto a las modificaciones sugeridas por la experiencia y por los dictados de la situación política. Ello ha concedido al MAA una gran versatilidad, clave de sus éxitos iniciales y de las innovaciones introducidas, aunque también explica porqué algunos de los elementos de mayor interés del MAA han podido ser eliminados con facilidad, al cambiar las actitudes y los intereses políticos.

\section{CUADRO 1}

\begin{tabular}{|l|r|r|r|r|r|r|r|r|}
\hline & 1985 & 1986 & 1987 & 1988 & 1989 & 1990 & 1991 & 1992 \\
\hline PALEONTOLOGIA & 0.43 & $\ldots$ & - & 1.64 & 0.5 & 3.04 & 2.95 & 1.8 \\
\hline PALEOLÍTICO & 0.49 & 0.5 & 0.5 & 0.5 & 2.5 & 7.9 & 3.9 & 4.1 \\
\hline NEOLITICO & 2.15 & $\ldots$ & $\ldots$ & 6.5 & 1.0 & $\ldots$ & $\ldots$ & 2.0 \\
\hline COBRE-BRONCE & 36.6 & 12.8 & 16.1 & 9.8 & 20.3 & 25.5 & 22.7 & 17.2 \\
\hline PROTOHISTORIA & 27.6 & 7.2 & 16.3 & 13.5 & 23.3 & 14.4 & 22.3 & 18.0 \\
\hline ROMANO & 21.5 & 5.6 & 16.0 & 8.0 & 13.0 & 5.2 & 22.6 & 9.8 \\
\hline MEDIEVAL & 9.09 & 6.9 & 5.2 & 6.2 & 9.7 & 2.6 & 6.7 & 3.5 \\
\hline SUBACUATICA & 0.18 & $\ldots$ & $\ldots$ & 1.0 & $\ldots$ & $\ldots$ & $\ldots$ & 0.4 \\
\hline $\begin{array}{l}\text { PINTURA } \\
\text { RUPESTRE }\end{array}$ & 1.07 & $\ldots$ & & & & & & \\
\hline
\end{tabular}

Presupuestos aproximados de los Proyectos de Investigación. Las cantidades están expresadas en millones de pts. Las del año 1985 están obtenidas de la "Memoria de Gestión" publicada en el Anuario de Arqueología Andaluza de dicho año. El resto procede de la sección "Fichas Técnicas", del volumen publicado en Huelva (Investigaciones, 1993). Estas últimas cifras deben incrementarse, especialmente para los años 1986 y 1987, ya que algunos proyectos no consignan los datos de todos los años, y además muchos proyectos fueron cerrados entre 1987 y 1988 y la mayoría de ellos no se presentaron en la citada reunión de Huelva.

Junto a todo ello no debe olvidarse que el apoyo político al Modelo se plasmó también en la concesión de unos presupuestos relativamente altos (Cuadro I), especialmente si los comparamos con los de otras Comunidades. En principio las cantidades destinadas a los Proyectos y a las Urgencias fueron globalmente simila- res, después la relación fue decantándose hacia estas últimas.

\section{III.1. La filosofía del modelo}

La idea inicial era luchar contra las concepciones derivadas de la historia del arte, tanto en su variante de "objeto antiguo" como en la de "belleza", pero sobre todo contra las positivistas que marcan como objetivo último la mera descripción de objetos, y que limitan de forma importante las posibilidades de la arqueología como instrumento de investigación histórica (6). Junto a ello destacaba una preocupación, al menos teórica, por la conservación del patrimonio excavado, y otra mucho más concreta por la difusión de los resultados, cortando con la costumbre de almacenar materiales sin estudiarlos que ha caracterizado tradicionalmente a la arqueología española. Clarificando la forma de alcanzar estos objetivos, el presidente de la Comisión A. Ruíz (1989) indicaba que el MAA procuraba sustituir la actuación por el proyecto, la matriz secuencial, excavación-conservación, por otra estructural y dialéctica y la difusión selectiva, por la difusión social.

La primera ruptura es el terreno donde el MAA tiene sus mejores éxitos. La formulación concreta, definida paulatinamente, consistió en diferenciar el Proyecto de las Actividades arqueológicas.

El Proyecto tiene que tener objetivos históricos claramente señalados, una programación plurianual y se concede por un máximo de seis años.

Las Actividades Arqueológicas Sistemáticas (Prospección superficial, Prospección con sondeos estratigráficos, Excavación sistemática, Estudio de materiales arqueológicos, documentación gráfica, actividades subacuáticas, restauración...) (7) se solicitan cada año. Para conce-

(6) La ideología personal de cada investigador tuvo escasa incidencia en el desarrollo inicial del modelo, como lo demuestra la variada composición de las distintas Comisiones de Arqueología.

(7) Orden de 28 de enero de 1985 , por la que se regula el otorgamiento de autorizaciones para la realización de actividades arqueológicas en la Comunidad Autónoma de Andalucía (BOJA de 15 de febrero de 1985) y Orden de 10 de Octubre de 1985 (BOJA de 12 de Diciembre de 1985). Sustituido recientemente por el Reglamento de Actividades Arqueológicas, aprobado por el Decreto 32/1993 de 16 de marzo (BOJA de 4 de mayo de 1993). 
derse tiene que estar aprobado el proyecto, pero la aprobación de este no presupone la de las actividades, para las que se exige la correspondiente memoria, demostrar que se cuenta con el equipo adecuado, haber entregado para su publicación un informe con los resultados de las actividades realizadas el año anterior, etc.

La segunda ruptura pretendía deshacer buena parte de los planteamientos erróneos sobre las diferencias entre investigación y gestión, que llevaba a tratar las excavaciones de urgencia como intervenciones de segundo orden por no estar dirigidas por un proyecto de investigación, y a considerar como algo ajeno al trabajo arqueológico la conservación y la restauración. Estos objetivos han estado lejos de alcanzarse (Salvatierra, 1994a).

La tercera ruptura, el conseguir romper con la difusión selectiva, es decir, la teoría de publicar "sólo lo importante" o "cuando los resultados estén completos", se procuró realizarla obligando, desde la propia Administración, a la presentación de los resultados de cada actuación a través de varias vías, con independencia de las propias a las que pudiera tener acceso cada investigador. Estas vías oficiales a partir de 1987 se estructuran en tres:

* Las Jornadas de Arqueología Andaluza, que se celebraban anualmente en el mes de enero (8) y donde todos los grupos que hubieran recibido autorización para la realización de actividades arqueológicas el año anterior debían exponer sus resultados. Las excavaciones de urgencia, con independencia del presupuesto invertido en las mismas, han estado en la práctica ausentes de estas.

* El Anuario Arqueológico de Andalucía que recoge en tres volúmenes la memoria de gestión preparada por cada Delegación Provincial, y los resultados de las actividades sistemáticas y de urgencia. Hasta el momento se han publicado los correspondientes a los años de 1985 a 1990.

* Las Memorias Finales de los Proyectos que deben realizarse tras un máximo de seis años de actividad de los mismos. Posteriormente este tipo de publicación se ha hecho extensiva a las grandes excavaciones de urgencia. Los primeros Proyectos finalizaron entre 1991 y 1992, y las memorias han sido solicitadas por la Dirección

(8) A partir de 1994 parece que va a procurarse que las mismas tengan lugar en el último trimestre del año.
General, habiendose interrumpido en consecuencia la mayoría de ellos.

Independientemente del mayor o menor éxito de esta política, no cabe duda de que se ha introducido un importante factor corrector a la búsqueda de una arqueología científica y orientada realmente a la reconstrucción de los procesos históricos, y a la conservación del Patrimonio Histórico. Los pasos dados para construir el modelo y los cambios introducidos en el mismo hasta llegar a la situación actual, es lo que procuraremos describir a continuación.

\section{III.2. 1984-1986. Las características iniciales}

El desarrollo del MAA comienza en 1984 con el Decreto de Creación de la Comisión Andaluza de Arqueología como órgano asesor de la política arqueológica de la Junta de Andalucía (9), la definición de la figura de los arqueólogos provinciales (desde ahora AP) y la publicación del Reglamento de Arqueología Andaluza (10).

La idea de que la investigación "pura" debía dirigir todo el proceso, se plasmó en la dependencia en que se situó a los AP de la Comisión de Arqueología, teniendo con esta encuentros periódicos en los que debían rendir cuentas de su actividad. Se trataba sin duda de una importante contradicción, ya que se situaba a un órgano administrativo jerarquizado, en cierta dependencia de uno asesor. Simultaneamente, y de modo paradójico, al AP se le asignó la función de controlar el correcto desarrollo de las excavaciones -incluidas, claro está, las de los miembros de la Comisión-, como inspectores de la Administración, con posibilidad de emitir informes negativos y recomendar la suspensión de permisos. El "control" recíproco se reveló muy pronto escasamente operativo y generador de conflictos, y así mientras la "inspección" cayó en desuso rápidamente, los "encuentros" se eliminaron en 1988.

(9) Decreto $248 / 1984$ de 25 de septiembre por el que se crearon las Comisiones Andaluzas de Bellas Artes (BOJA de 19 de octubre de 1984). Entre ellas la de Arqueología. Tras la aprobación de la Ley del Patrimonio de Andalucía de 1991, toda la normativa está readecuándose: se ha creado el Consejo Andaluz del Patrimonio Histórico, constituyéndose en su seno las Comisiones Andaluzas de Bienes Culturales (Decreto $4 / 93$ de 26 de enero, BOJA 18 de febrero de 1993 por el que se aprueba el Reglamento de Organización Administrativa del Patrimonio Histórico de Andalucía).

(10) Ibidem. nota 8 
A lo largo de todo el periodo y después, se fomentan reuniones entre los arqueólogos provinciales, con el fin de intercambiar experiencias, homogeneizar en lo posible criterios de actuación, y que ellos mismos pudieran, a partir de su práctica, proponer elementos de desarrollo del modelo de gestión.

Aunque en esos momentos iniciales aún no es evidente, la propia configuración del MAA genera un divorcio entre investigación y gestión, sin que se prevean realmente mecanismos de integración. En gran medida el problema reside en que, debido a la exclusiva orientación investigadora de casi todos los arqueólogos, no se desarrolla realmente una teoría de la gestión (por ejemplo sobre acondicionamiento y uso de yacimientos), o sobre la conservación. Prueba de ello es que el único mecanismo que se establece está dirigido a tratar de integrar los registros de las urgencias en la investigación, y consiste simplemente en asegurar la publicación de los informes provisionales de tales excavaciones.

No obstante, todas estas medidas indican que la Administración andaluza estaba realizando un serio esfuerzo para crear estructuras y normas capaces de abordar la cuestión del $\mathrm{Pa}$ trimonio Arqueológico en sus diversas vertientes.

\section{III.3. 1987-1988. El desarrollo práctico}

A.- Las Decisiones. Como hemos dicho el Modelo no se consideró terminado tras la creación de la estructura administrativa y asesora, sino que desde el principio fueron introduciéndose modificaciones en el funcionamiento, a medida que la experiencia y la discusión aclaraba o ampliaba los conceptos iniciales. Entre las decisiones más significativas tomadas en este periodo cabe citar:

a.- Reducción del número de Proyectos de Investigación, al afinarse las valoraciones de los trabajos realizados, y eliminar aquellos proyectos carentes de objetivos claros, de equipos solventes o suficientemente amplios, o de metodologías adecuadas.

b.- Exigencia de integración de todas las formas de intervención (especialmente prospección y estudio de materiales) y drástica reducción de las excavaciones. c.- Limitación a un Proyecto por grupo de investigación en cada periodo, con una sóla excavación sistemática, y con la obligación de alternar anualmente la excavación y el estudio de los materiales.

d.- Incremento constante del presupuesto para las excavaciones de urgencia, que en 1988 supera claramente al de "investigación". Esto permite contratar a arqueólogos para su realización, acabando con el voluntarismo dominante hasta entonces.

e.- Hasta 1987 las urgencias habían incluido pocas excavaciones urbanas, pero su número irá incrementándose rápidamente.

B.- Problemas y debates. El desarrollo del modelo, sus indudables éxitos iniciales en diversos aspectos, y los relativamente elevados presupuestos, comparados con lo que siempre ha sido tradicional en nuestro país, produjeron una considerable ampliación del ámbito de intervención, lo que a su vez hizo que se manifestasen diversos problemas, de manera que las medidas que en un principio suponían un gran salto adelante, en 1988 van siendo insuficientes. Entre las cuestiones que ahora se plantean, y que mayor proyección tendrán en los años siguientes pueden señalarse:

a.- El problema de la conservación y la restauración en los yacimientos arqueológicos. En principio aquellos sobre los que deberían ejercerse tales actuaciones son por un lado los incluidos en los Proyectos de Investigación y por otro aquellos que estuviesen en peligro por cualquier causa. Por lo que se refiere a los primeros, la administración, excepto en unos casos muy concretos, no dedica cantidades complementarias a estas cuestiones, ya que insiste en que la conservación debe ir incluida en el planteamiento de la actividad. Pero desde el punto de vista de los investigadores, los presupuestos resultan demasiado ajustados para que sea posible dedicar parte de los mismos a esas tareas. Paralelamente, los destinados globalmente para la protección y la conservación, a los que hipotéticamente podrían tener acceso los AP, son exclusivamente para restauraciones arquitectónicas, en las que la arqueología no juega ningún papel. En esta situación los AP no disponen de dinero para intervenir, ni de medios para lograr que los investigadores inviertan en conservar. A ello se 
agrega que tampoco hay acuerdo sobre los criterios a seguir.

En este marco, entre algunos de los AP se inicia la construcción de un discurso teórico, según el cual la contradicción del sistema debía resolverse mediante la eliminación de las actuaciones de "investigación pura", ya que la Comunidad no podía permitírselas, y porque las actuaciones orientadas por la gestión serían suficientes para cubrir las necesidades de los Proyectos que, para los mas radicales, deberían ajustarse a las necesidades marcadas desde la propia administración.

b.- La falta de clarificación de la situación laboral de los arqueólogos provinciales. Hay una evidente indecisión de los responsables políticos acerca de la conveniencia o no de crear un cuerpo de funcionarios Conservadores del $\mathrm{Pa}$ trimonio y de ampliar la plantilla para cubrir las necesidades en aumento. En este marco empieza una tensa discusión a cerca de si es posible que se desarrolle una auténtica política de intervención, sobre todo en el campo de la protección y la conservación, sin que ello se plasme en la propia estructura administrativa.

c.- Los conflictos creados en las ciudades ante el incremento en el número de intervenciones. Estos surgen ante actuaciones en solares que provocan la parálisis de grandes obras, unas de otras administraciones públicas (por ej. el aparcamiento que desea construir el Ayuntamiento de Córdoba en la Avenida de Gran Capitan (11)) y otras de empresas privadas (como el plan de urbanización del cerro de Los Alijares de Granada (12)). Sólo los mayores llegan a alcanzar cierta relevancia y se salvan con negociaciones al máximo nivel; muchos otros se resuelven a nivel local o, simplemente, se cierran los ojos a la destrucción, tras valorar el costo del enfrentamiento en comparación con los elementos aparecidos. Esta situación manifiesta la contradicción existente entre las tendencias que desean la conservación del patrimonio, considerado como un bién en sí, y las del desarrollismo y la especulación urbanística.

(11) Se trata de una de las avenidas centrales de la ciudad, que desemboca en el barrio antiguo. Tras la decisión de que no se construyese, aceptada por el Ayuntamiento, este último procedió a enterrar los restos.

(12) Se encuentra por encima de La Alhambra, condicionando la visión de esta. En la actualidad ha sido objeto de un proyecto de Reforma Interior dentro del Plan Especial del conjunto (Plan Especial, 1986). d).- La destrucción de patrimonio producida por el aumento de restauraciones en castillos y otros edificios históricos sin que se realice una investigación arqueológica previa. A lo que se añade el incremento de las actuaciones en los mismos de Escuelas Taller, que sólo en ocasiones han contado con la dirección de un arqueólogo, y nunca con equipos especializados, pero sí con presupuestos mucho más elevados que los de cualquier excavación sistemática, y sin que se prevea el estudio del material obtenido.

En este marco, la evolución de la situación permite observar ya en 1988 dos debates claves. Uno, sobre los problemas que genera la intervención. El segundo, centrado en el tema no menos crucial de quién debe asumir el coste de dicha intervención.

Respecto al primero se advierten dos posturas claras. Por un lado están quiénes apuestan por la gestión y la intervención consecuente, y que juegan al máximo con las posibilidades de actuación, dosificando las posibilidades de presión según los casos y coyunturas (13), y paralelamente procuran implicar en todo el proceso de actuaciones a los distintos grupos de investigación que trabajan en su zona.

La segunda línea, aunque habría que hablar más bien de extremo opuesto, la representan los que optan por un tratamiento "político", asumiendo que la arqueología de urgencia es conflictiva y que su papel, como miembros de la administración, es reducir al máximo el conflicto, por el medio que sea, incluso no interviniendo en absoluto.

Por lo que se refiere al segundo debate, para unos es la administración la que debe subvencionar las intervenciones, y sólo debe costearla el particular cuando este exija que su solar se excave inmediatamente, sin esperar "turno" (14). Pero en cualquier caso los honorarios del ar-

(13) Así se tiene en cuenta la capacidad económica de quién construye (ya que los promotores van desde la propia administración o las grandes empresas, hasta los particulares), el objetivo de la construcción (gran bloque de pisos para la venta, casa individual propia, etc), la importancia arqueológica de la zona teniendo en cuenta intervenciones anteriores en la proximidades, etc.

(14) Las excavaciones se efectúan a medida que va llegando el presupuesto, y según va solicitándose por el promotor o paralizándose la obra ante la aparición de restos. El retraso en la provisión de fondos o el agotamiento del presupuesto disponible en un momento dado, conduce al establecimiento de una "cola" y por tanto de un "turno". En numerosas ocasiones, el ahorro de tiempo puede compensar al promotor, que decide costear la excavación. 
queólogo debe abonarlos siempre la administración, reservándose así el derecho de designar al especialista.

Por el contrario, para otros, celosos protectores de la administración, todos los costes debe pagarlos el promotor. Pero éste obtiene así una fuerza considerable a la hora de contratar al arqueólogo, la misma que el AP pierde para controlar las actuaciones de aquel. Esta situación se produce en otras Comunidades, como la de Madrid, habiendose señalado repetidamente sus peligros (Domínguez y otros, 1994).

Esta compleja cuestión genera simultaneamente divisiones entre otros colectivos. Para algunos de los directores de Proyecto de Investigación, el control de las urgencias se convierte en una cuestión de primer orden. En teoría esta preocupación estaría producida por la necesidad de garantizar la calidad científica y por el problema de cómo incorporar el registro de las excavaciones de urgencia a los proyectos de investigación. Pero en bastantes casos no debe olvidarse que el control de las urgencias supone el control, aunque indirecto, de un importante presupuesto y la posibilidad de que los arqueólogos sin trabajo remunerado, integrados en el grupo de investigación, cobren y se mantengan (Salvatierra, 1994b).

Esa misma circunstancia, que produce el surgimiento en cada provincia de un colectivo de arqueólogos que viven de las excavaciones de urgencia, da lugar entre muchos de ellos a la idea del arqueólogo profesional, realizándose diversos intentos de articulación entre los colectivos existentes. No obstante, fracasan totalmente ante las profundas diferencias existentes entre ellos mismos, entre las que ocupa un lugar importante la división entre las dos opciones respecto al pago arriba señaladas.

C.- La Especificidad Medieval: A lo largo de toda esta fase, en la Comisión de Arqueología figuran dos medievalistas, que realizarán un notable esfuerzo para elevar el nivel del trabajo que se hacía en Andalucía, equiparándolo con las corrientes europeas actuales, línea no siempre bien entendida. De esta forma, Andalucía es la primera Comunidad Autónoma donde la arqueología medieval recibe una atención similar a las áreas tradicionales, factor que aún está lejos de producirse en algunos otros territorios peninsulares.
Por otra parte, la mayoría de las intervenciones de urgencia tienden a concentrarse de modo natural en los núcleos urbanos, y casi todos estos proporcionan restos medievales, con lo que las excavaciones de esta época se multiplican. Pero esta situación de aparente privilegio adquiere muy pronto aspectos preocupantes. Como hemos dicho, el incremento en los presupuestos de las urgencias hace que estas se conviertan en la fuente de recursos de los arqueólogos recién terminados, en la mayoría de los casos sin conocimientos ni interés por la época medieval y sus problemas (Salvatierra, 1994a y b). lo que lleva en algunas zonas a la destrucción sistemática de los restos postclásicos curiosamente siempre "mezclados", para llegar a "los niveles in situ" -siempre romanos o anteriores- o, como "mal menor", a una más que deficiente documentación del registro. La sorprendente falta de niveles medievales en la mayoría de las ciudades andaluzas, tal y como podría deducirse de los resúmenes contenidos en los volúmenes del Anuario Arqueológico de Andalucía de estos años, nos eximen de profundizar en la cuestión.

\section{III.4. 1988. La crisis}

A principios de 1988 el modelo parece aún claramente positivo. La mayoría de los problemas que surgen pueden achacarse a dificultades puntuales, o a errores subsanables. Por lo que respecta a las contradicciones existentes, pese a la gravedad de algunas, la continuidad y profundización del debate parecen garantizar que se encontrarán soluciones adecuadas. La mas grave de estas contradicciones es el falso dilema entre conservación y desarrollo, que conduce al conflicto. Los que surgen a lo largo de estos primeros años se salvan negociando y con mayor o menor coste, lo que da la impresión de que no hay situaciones insalvables. Pero la cuestión es en realidad un problema estructural, irresoluble en los parámetros en que está planteado.

La situación que finalmente precipitará la crisis arranca con la apariencia de un conflicto más en Octubre de 1987, al aparecer unas espectaculares murallas en la plaza de La Marina, en Málaga, y que inmediatamente empiezan a ser demolidas por el ayuntamiento (15). La Dirección General paraliza las obras y se realiza

(15) Málaga, Diario Sur 9-X-1987; Diariol6 9-X-1987 
una amplia intervención arqueológica (16). Tras comprobar la importancia de lo aún existente se tratará de llegar a un acuerdo entre las pretensiones municipales de construir un aparcamiento y la necesidad de conservar las importantes murallas. El asunto se complica, produce enfrentamientos dentro del partido gobernante y finalmente el empeño en la conservación cuesta el puesto al Director General de Bienes Culturales, D. Bartolomé Ruíz, principal ideólogo e impulsor del MAA hasta ese momento (17).

De esta forma queda de manifiesto que la mayor debilidad del Modelo es que, en realidad, este no responde a una voluntad política expresa y asumida por los grupos políticos, ni a un desarrollo social profundo, sino que ha sido más bien resultado de un dejar hacer en un tema sobre el que el desconocimiento, la indiferencia y la ignorancia de la mayoría de los dirigentes políticos eran totales. Pero en el momento en que se hace evidente que la conservación puede chocar no ya con los fuertes intereses económicos que rodean a toda intervención urbana, sino con los intereses particulares de la administración local, los grupos políticos no dudarán en actuar como se ha visto en el caso de Málaga, obviando la legislación existente a este respecto, no sólo la promulgada a nivel nacional, sino la propia de Andalucía que, en el caso del Patrimonio Histórico, fue aprobada prácticamente por unanimidad de todos los grupos políticos, los mismos que gobiernan los ayuntamientos. A partir de ese momento, se hace evidente que la batalla por conservar restos arqueológicos sólo podrá librarse en circunstancias muy específicas.

\section{III.5. 1989-1993. Hacia un nuevo modelo}

La sustitución de D. Bartolomé Ruíz se procura que sea lo menos traumática posible, y él

(16) Málaga, Diario Sur 16-IV-1988

(17) Málaga, Diario Sur 26-V-1988. Tras ello, y pese a las tajantes intrucciones en contra de la Consejería de Cultura, el Ayuntamiento siguió destruyendo la muralla, realizando el trabajo por la noche y tras cubrir esta con un toldo para que no se advirtiese. Ante las denuncias, el Sr. alcalde D. Pedro Aparicio, afirmó que nada sabía del asunto, y que iba a "abrir una investigación" (Málaga, Diario Sur 22-I-1989) que parece que aún no ha concluido. Por su parte la Dirección General de Bienes Culturales abrió expediente sancionador al Ayuntamiento (Málaga, Diario Sur 24-I-1989). mismo realiza gestiones para impedir la dimisión en bloque de la Comisión de Arqueología, mientras se garantiza por parte de los responsables políticos, en especial por el nuevo Director General, D. José Guirao, la continuidad del modelo. No obstante, muy pronto comenzará a desarrollarse un nuevo programa, y después, tras las elecciones autonómicas de 1990), se pone de manifiesto que el nuevo equipo de gobierno tiene planteamientos muy diferentes de los seguidos hasta entonces por lo que respecta al $\mathrm{Pa}$ trimonio Arqueológico.

Algunos de los nuevos factores han supuesto en la práctica la liquidación de varios de los elementos más avanzados del MAA, y el inicio del camino hacia un nuevo modelo, aunque los pasos dados son todavía titubeantes y contradictorios.

\section{A.- La reconversión de la Comisión de Arqueología}

Uno de los elementos más originales del Modelo era la Comisión Asesora, formada por arqueólogos independientes y a la que en la práctica se le reconocían amplias competencias, entre ellas el derecho -y prácticamente la obligación- de debatir todas las cuestiones referentes a la arqueología. El propio desarrollo del modelo le fue restando paulatinamente funciones (intervención en monumentos, urgencias, etc), pero es en este momento cuando se produce un cambio radical de su papel.

En los primeros años, ante cada conflicto se convocaba a la Comisión al lugar y, tras el informe de la misma, se producía la negociación o la resolución, según los casos, del problema. Ahora se prescinde de la Comisión para estas tareas, asumiendo el Director General el tema a partir del informe del arqueólogo provincial correspondiente o, en todo caso, con asesores elegidos al margen de la Comisión. Con ello, en teoría, la situación se vuelve menos crispada, y aparentemente el desgaste político de la Comisión de Arqueología es menor, puesto que ya no es utilizada como "ariete" frente a las administraciones locales. Además se refuerza el papel de la legislación como elemento decisivo en la actuación, con independencia de la valoración científica de los restos de que se trate.

Pero con la intervención de la Comisión la acción protectora se apoyaba en el dictamen de un grupo de especialistas seleccionados por su 
capacidad con anterioridad al surgimiento de cualquier problema, lo que proporcionaba fuerza a dicho dictamen. Ello dificultaba la adopción de medidas exclusivamente "políticas", que por lo general suelen implicar la destrucción final de los restos de que se trate. Con el nuevo sistema, por el contrario, esa limitación no existe y todo depende de la voluntad o fuerza política que en cada momento tenga el Director General.

Esta forma de operar se ha extendido a todos los temas, de forma que la Comisión ya no es en la práctica un órgano de debate y asesoramiento de la política arqueológica. Sus casi únicas funciones son informar sobre los Proyectos de Investigación, recomendar la concesión de autorizaciones para los diferentes tipos de intervención y proponer la distribución de subvenciones. La única decisión de cierta relevancia en estos años ha sido la creación, con cargo al presupuesto de excavaciones, de becas orientadas a la formación de arqueólogos en nuevos métodos y técnicas aplicables al patrimonio, cuya primera convocatoria es de 1991. Factor sin duda positivo, aunque no se haya conseguido que dichas becas sean reconocidas a ningún nivel por la Consejería de Educación de la propia Junta de Andalucía.

\section{B.- El nuevo rumbo de la gestión}

Tras las numerosas dudas del periodo anterior, la Administración opta por crear el cuerpo de funcionarios de Conservación del Patrimonio, único para arqueólogos, arquitectos, historiadores del arte y etnólogos, retomando así los planteamientos, en el aspecto de unir los cuatro campos, del Plan General de Bienes Culturales. Las primeras oposiciones se realizan entre 1990 y 1991 y las segundas entre 1993 y 1994, con 15 plazas convocadas en total. Tras las mismas la división de Arqueología está integrada por seis personas (18). Este escaso número, y la compleja situación por la que atraviesa la arqueología andaluza, hacen que este factor resulte secundario en los momentos actuales, aunque posiblemente será muy importante a largo plazo.

(18) De ellas cinco por oposición y una sexta incorporada desde la Diputación de Málaga, donde se había creado un puesto equivalente.
Aparte de esta cuestión, es posible advertir en estos años dos cambios sucesivos de política, uno tras la crisis, y el segundo a partir de 1992.

El primero puede considerarse como la respuesta inicial de la Administración ante el problema del conflicto urbano, y tiene dos aspectos, la conservación y la documentación.

a.- La conservación tras la excavación resulta para la administración andaluza el auténtico foco de problemas, por el enfrentamiento con las administraciones locales, cada vez mas frecuente aunque raramente se reconozca públicamente y la falta de actuación se justifique por la falta de recursos para adquirir solares, la dificultad de integrar ruinas en ciudades, etc. En muchos casos puede discutirse efectivamente la racionalidad de conservar o no, pero lo grave es que exista una decisión política general de no hacerlo si ello supone un conflicto, como lo demuestra el caso de la Estación de Cercadillas de Córdoba.

En esta última apareció uno de los conjuntos monumentales romano-tardíos más impresionantes de la Península Ibérica y posiblemente de Europa (Márquez y otros, 1992) (19). Por otro lado, la destrucción del gran conjunto ha llevado a considerar como muy secundario el total arrasamiento, sin apenas documentarlo, del arrabal islámico (al parecer del siglo XII) ubicado por encima del mismo, destrucción de la que apenas se ha dicho una palabra, y que continúa hoy con barrios islámicos de la periferia cordobesa.

b.- Si bien no se va a conservar, se postula que sí es preciso documentar todo lo posible. Esta política se llevará a cabo mediante el incremento de los presupuestos de las excavaciones de urgencia, al tiempo que aparentemente se da un mayor respaldo a los AP para que intervengan en núcleos urbanos, o al menos esto es lo que puede deducirse para varias ciudades a la luz de los informes publicados en los anuarios de arqueología del periodo 1988-1991.

Ante esto la actitud de los AP será diversa, observándose en general dos tendencias. Para unos, el nivel mínimo es la exigencia de documentar completamente antes de que se autorice la construcción, y debe procurarse ir al máximo

(19) Es preciso advertir que todos los grupos políticos del ayuntamiento, gobernado por Izquierda Unida, apoyaron la destrucción, cuando ya era de sobra conocida su enorme importancia. 
de intervenciones. Para otros, sólo habría que documentar si los restos fuesen muy importantes y en consecuencia sólo habría que hacer el "seguimiento" de las obras.

Las diferencias se justifican en base al argumento de que cada ciudad presenta condiciones diferentes. El Director General parece tomar la decisión de ser muy pragmático, y dejar que el AP local, que es quién mejor conoce su zona, actúe según sus criterios. Esta opción, en principio perfectamente razonable y que parece desarrollar hasta cierto punto la situación anterior, provoca importantes disfunciones y tampoco soluciona la cuestión de las situaciones conflictivas ante grandes obras, dado que o bien el arqueólogo de turno procura minimizar la importancia de lo hallado o más generalmente los ayuntamientos se niegan a colaborar, haciéndose necesaria en uno y otro caso la intervención de la Dirección General.

De nuevo, Cercadillas sirve de magnífico ejemplo de esta política: falta de información inicial de la Delegación Provincial a la Dirección General sobre la importancia de los restos, paralización inmediata cuando al fin se conoce esta, trabas y objeciones del Excmo. Ayuntamiento y fuerte inversión a lo largo de prácticamente dos años, para autorizar después la construcción de la estación (20). No se adoptó ninguna medida sancionadora ni contra el AP, ni contra el Delegado Provincial, ambos responsables de la situación creada.

En consecuencia, tanto la destrucción del yacimiento de Cercadillas, como la amplia inversión realizada desde la Dirección General para documentar la fase romana, no son sino la clara expresión del "Nuevo Modelo", en lo positivo y en lo negativo, y lo que demuestra más cabalmente el agotamiento del "Antiguo".

El segundo cambio de política se produce a partir de 1992. Consiste básicamente en la pérdida de autonomía de los AP a la hora de actuar; progresivamente la Dirección General va

(20) Se ha afirmado repetidamente que el proyecto debía realizarse porque afectaba al AVE y esta era una "cuestión de Estado". El argumento es inaceptable ya que la Conservación del Patrimonio Histórico es un precepto constitucional (Art. 46) y está por encima de los intereses del gobierno de turno, por fundamentales que tales intereses sean para sus objetivos electorales. Pero es que ni siquiera eso es cierto, ya que en la zona lo que va es la estación de Córdoba y fundamentalmente la playa de vías de la misma. asumiendo todas las decisiones en relación a la paralización de obras y sobre las excavaciones a realizar y el momento de ejecución de las mismas. Hasta cierto punto con ello parece pretenderse que la información llegue realmente a la Dirección General en tiempo y forma, y que se eviten situaciones como las comentadas más arriba. Pero es imposible desligar esta situación de la falta de liquidez económica de la Junta de Andalucía (21), que deja casi sin presupuesto a la arqueología durante 1992 y 1993. Por ello no es de extrañar que, aunque se crea una normativa interna dirigida a agilizar las gestiones, el cambio suponga un mayor retraso a la hora de actuar y de que lleguen las escasas subvenciones aprobadas.

En esta situación, y con el precedente de Cercadillas, el problema del control de la calidad de las intervenciones de urgencias a que nos hemos referido antes, sigue agravándose y la arqueología urbana degenera, aunque en las Jornadas de Arqueología Andaluza de Huelva (Enero 1993) se decidiese que estaba en perfectas condiciones y que sólo había pequeños problemas.

Por otro lado, ninguno de estos cambios de política ha significado que ahora se vaya a intervenir en los ámbitos sobre los que antes no se actuaba, carencias que hemos descrito como los principales defectos del sistema en las primeras etapas. En ese aspecto la situación seguirá igual, es decir, la arqueología sigue ausente de los monumentos en restauración, llegándose a extremos como el de que apenas pueda intervenirse en la Alcazaba de Málaga, pese a las importantes remociones de tierra. Por otra parte, el control de los AP sobre las Escuelas Taller sólo se produce en algunos casos.

\section{LAS TENDENCIAS ACTUALES}

La transformación del MAA ha coincidido en el tiempo con la mencionada falta de liquidez de la Junta de Andalucía, pero también con el fin del periodo de seis años de duración de la mayoría de los Proyectos de investigación y,

(21) Esta, con independencia de las afirmaciones de los políticos de turno, parece haber estado causada más por el endeudamiento derivado de la campaña de propaganda y relumbrón denominada EXPO92, que por la manida "crisis económica". 
como se señaló al principio, antes de optar a otro es necesaria la presentación y aprobación de la memoria final. Ello ha concedido a la administración un amplio respiro que le ha permitido reducir drásticamente los presupuestos de arqueología de investigación, sin que haya habido excesivas quejas. El que la Comisión haya visto limitadas sus competencias efectivas, y que la política seguida haya cambiado, tampoco ha producido mayores problemas, con la excepción de un par de dimisiones, por otra parte muy separadas en el tiempo.

Por lo que respecta a la gestión, está completándose el marco legislativo, con nuevas normas, documentos de trabajo y proyectos de reglamentos, como el de Actividades Arqueológicas al que ya hemos hecho referencia (22), con novedades como la exigencia de que los equipos de investigación cuenten con un arquitecto. Puesto que he tenido ocasión de referirme a este texto recientemente, no volveré ahora sobre el mismo (Salvatierra, en prensa).

Están en fase de borrador los Reglamentos de Protección y Restauración. Este último, según manifestaciones del Director General, incluirá la obligación de que en toda restauración actúe un arqueólogo. Por último está la decisión de impulsar la informatización del registro arqueológico.

En otro plano, a principios de 1993 se sacaron a concurso los primeros proyectos urbanos, creados por la Dirección General, dirigidos a poner orden en el desbarajuste existente en las principales ciudades, tratando de forzar una coordinación entre los arqueólogos que realizan las urgencias, y procurando que se establezcan programas que racionalicen las investigaciones necesarias. Esta cuestión puede relacionarse con la teoría de que la administración debe indicar a los investigadores cuales son sus prioridades, lo que sin duda es positivo, aunque depende de la estructura, fines, medios y límites con que se haga. Por el momento el proyecto urbano y las urgencias a realizar, no parecen tener demasiada relación entre sí, y por lo que sabemos en cada ciudad se está enfocando desde una perspectiva diferente, lo que quizá sea enriquecedor, pero no sabemos si efectivo. Parece claro que cuando menos puede darse un problema de competencias entre las del director del proyecto y del grupo de investigación que lo

(22) Ibidem. nota 8 asume, y las del AP correspondiente, por no mencionar las que se reserva para sí la propia Dirección General.

En conclusión, en el terreno práctico, se ha creado un nuevo modelo de funcionamiento en el que el AP pasa de ser un agente activo en la toma de decisiones, a mero informante y emisor de propuestas, mientras que la Comisión Asesora pierde protagonismo. Se trata por tanto de un aumento del centralismo y la jerarquización (también burocratización) administrativas. Pero también se han tomado algunas decisiones que pueden conducir a que de nuevo las urgencias pasen a estar controladas por los grupos de investigación, superponiendose al AP. Parece evidente que esto es contradictorio entre si y a su vez con el desarrollo de un cuerpo de funcionarios de Conservación del Patrimonio.

La confusión sobre la situación existente se refleja, por otro lado, en los intentos que se producen en cada provincia por crear un 'modelo' propio, que van desde unas donde el Delegado Provincial prácticamente asume las competencias del AP aprovechándose de la debilidad de este, a otras donde se intenta recuperar la antigua idea de la Comisión Provincial que asesore al AP, o los nuevos intentos de articulación mediante empresas, de los que la universidad tampoco está ausente. En suma, el modelo no está definido, ni la administración tiene claro hasta dónde o cómo debe intervenir. Habrá que esperar a que pase la actual situación económica y tengan lugar las elecciones autonómicas previstas para el verano de 1994, para ver cual es realmente la estructura que se busca y si se intenta una nueva línea, o los actuales funcionarios quedan como "cuerpo a extinguir".

Por ahora sólo podemos concluir que el MAA de los años ochenta ha finalizado, y que estamos en un periodo de transición hacia uno que será bastante diferente. El único elemento que permite ser "optimista" es el hecho de que al menos aún existe en Andalucía cierta preocupación por desarrollar una Política Arqueológica, aunque esta en los últimos años no haya sido precisamente satisfactoria.

\section{BIBLIOGRAFÍA}

Acien Almansa, M. (1994): "Política y arqueología ¿dependencia?". Coloquio Problemas en Arqueología Medieval (Jaén 1993). Arqueología y Territorio Medieval, I: 67-74. Jaén. 
Arqueologia de las ciudades modernas superpuestas a las antiguas (Zaragoza 1983). Ministerio de Cultura. Madrid, 1985.

Arqueología del País Valenciano. Panorama y perspectivas (Alicante 1983). Universidad de Alicante, 1985.

Arqueología en Alicante 1976-1986. Instituto Juan Gil-Albert. Alicante, 1986.

Arqueologia urbana en Zaragoza (1984-1986). Ayuntamiento de Zaragoza. Zaragoza, 1986

Bl.Asco, G. y VAl.1.E. J. M. (1992): “Arqueología de gestión "versus" arqueología de investigación: El caso Madrid como paradigma". I Reunión de Arqueología Teórica. (Santiago de Compostela 1992). Actas distribuidas en fotocopias.

Dominguez, R. M.; Fernandez, J. L.; Herce, M.; Mena. SANCH, M. y Presas, M. (1994): “Empresas de arqueología y arqueología urbana: Investigación, negocio, profesión". Coloquio Problemas en Arqueología Medieval (Jaén 1993). Arqueología y Territorio Medieval, I: 83-91. Jaén.

Els enterraments del Parc de la Ciutat i la problemàtica funerària de Tàrraco. Memòries d'excavació, 1. Taller Escola d'arqueologia, Tarragona. 1987.

Homenaje a Luis Siret (1934-1984) (Cuevas de Almanzora 1984). Junta de Andalucía. Sevilla. 1986

Investigaciones Arqueológicas en Andalucia 1985-1992. Proyectos. Junta de Andalucía. Huelva, 1993.

La Plaza de la Seo. Zaragoza. Investigaciones histórico arqueológicas. Ayuntamiento de Zaragoza. Zaragoza, 1989

Márouez, C.; Marfil, P. e Hidalgo, R. (1992): "El complejo monumental tardorromano de Cercadilla". L'Africa Romana, Atti del IX convegno di studio. Sassari: 1039-1050.

Martínez Navarrete, M. I. (1989): “Una revisión crítica de la prehistoria española: La Edad del Bronce como paradigma". Siglo XXI. Madrid

Peral., C. (1994):“La arqueología urbana en Málaga (1986-1992): Una experiencia a debate". Coloquio Problemas en Arqueología Medieval (Jaén 1993). Arqueología y Territorio Medieval, I: 75-82. Jaén.

Plan Especial de Protección y Reforma Interior de La Alhambra y Alijares. Junta de Andalucía. Granada, 1986.
Primeras jornadas de arqueologia en las ciudades actuales (Zaragoza, enero 1983). Ayuntamiento de Zaragoza. Zaragoza 1983

RIt:RA. M. (1994): "Plancamiento urbanístico, promoción inmobiliaria y arqueologia involuntaria". Coloquio Problemas en Arqueologia Medieval (Jaén, 1993). Arqueología y Territorio Mediéval. I: 93-99. Jaén.

Rodriglle., A.: San Mil.aN. M. J.: Sanchez, J. M.; Chapa, T.: Martinez Navarre 11, M. I. y Rliz Zapa. TERo, G.(1993): "Análisis bibliométrico de Trabajos de Prehistoria: Un chequeo a la Prehistoria española de las tres últimas décadas". Trabajos de Prehistoria, 50: 11-37. Madrid.

Ruiz, A. (1989): "De las arqueologías a la arqueología". En 1978-1988. Andalucía: Diez años de Cultura. Junta de Andalucía. Sevilla: 9-17.

Ruiz, A.; Molinos, M. y Hornos, F. (1986): “Arqueología en Jaén. (Reflexiones desde un Proyecto arqueológico no inocente)". Diputación de Jaén. Jaén

Sal.vatierra, V. (1990): "Cien años de Arqueología Medieval. Perspectivas desde la periferia: Jaén". Universidad de Granada.

- (1994)a: "Arqueología urbana: Investigación y Gestión. La situación en Andalucía”. Coloquio Problemas en Arqueología Medieval (Jaén, 1993). Arqueología y Territorio Medieval, I: 75-82. Jaén.

- (1994)b: "El Patrimonio arqueológico urbano". Curso de Conservación del Patrimonio Arqueológico (Sevilla 1992). Instituto de Conservación del Patrimonio. Sevilla: 20-29.

- (en prensa): "El nuevo Reglamento de Actividades Arqueológicas de la Junta de Andalucía". Boletín del Instituto de Estudios Giennenses. Jaén.

Salvatierra, V.; Castillo, J. C. y Castillo, J. L. (1992): "Arqueología urbana e historia. El caso del Jaén islámico". I Coloquio Hispano-Italiano de Arqueología Medieval (Granada 1990). Patronato de La Alhambra. Granada: 109-122.

Velasco, F. (1992): “Un modelo de gestión a partir de la declaración de zonas arqueológicas amplias: el caso de Madrid". Jornadas Internacionales de Arqueología de Intervención. San Sebastián: 75-83. 